\title{
Plant Robot for At-Home Behavioral Activation Therapy Reminders to Young Adults with Depression
}

\author{
ASHWIN SADANANDA BHAT, University of Twente \\ CHRISTIAAN BOERSMA, LEDLoket \\ MAX JAN MEIJER, Microsoft Research \\ MAAIKE DOKTER, XABLU \\ ERNST BOHLMEIJER and JAMY LI, University of Twente
}

\begin{abstract}
Adolescents with depression who participate in behavioral activation therapy may find it hard to be motivated to perform tasks at home that their therapists recommend. We describe the initial design and usability evaluation of a home device ("PlantBot") that could be used to remind young adults with depression at home of their behavioral activation therapy-related tasks. The prototype features electronics in a two-layer base, with a fake plant on top and supported using the Amazon Echo voice agent. We use an online panel study to evaluate the usability of our system with youth with past depression $(N=30)$. Initial findings highlight the device's usability, potential benefit, and attractiveness of the plant component, as well as multiple improvements to be made.
\end{abstract}

CCS Concepts: • Human-centered computing $\rightarrow$ Interface design prototyping; Accessibility technologies; Usability testing; • Social and professional topics $\rightarrow$ Adolescents;

Additional Key Words and Phrases: Behavioral activation therapy, depression, plant robot, voice agent, Internet of Things, Amazon Echo, young adult, adolescent

ACM Reference format:

Ashwin Sadananda Bhat, Christiaan Boersma, Max Jan Meijer, Maaike Dokter, Ernst Bohlmeijer, and Jamy Li. 2021. Plant Robot for At-Home Behavioral Activation Therapy Reminders to Young Adults with Depression. ACM Trans. Hum.-Robot Interact. 10, 3, Article 20 (July 2021), 21 pages.

https://doi.org/10.1145/3442680

\section{INTRODUCTION}

Depression is a serious mental health issue that affects adolescents and adults globally. According to the World Health Organization [50], depression is characterized by sadness, feelings of low selfworth, guilt, tiredness, low motivation, and poor concentration. Depression and anxiety affect $25 \%$ of the population in Europe each year, with up to $74 \%$ of diagnosed cases of depression having had their first onset before the age of 24 years [39]. Depression is a major suicide risk, which is one of the leading causes of death among adolescents [70]. According to the Centers for Disease Control

The majority of this work was performed when all authors were at the University of Twente.

Authors' addresses: A. Sadananda Bhat, C. Boersma, M. J. Meijer, M. Dokter, E. Bohlmeijer, and J. Li, University of Twente, PO Box 217, 7500 AE Enschede, The Netherlands; emails: ashwinbhat712@gmail.com, boersmachristiaan@gmail.com, maxmeyer@live.nl, maaikedokter@hotmail.com, e.t.bohlmeijer@utwente.nl, jamy@ryerson.ca.

Permission to make digital or hard copies of all or part of this work for personal or classroom use is granted without fee provided that copies are not made or distributed for profit or commercial advantage and that copies bear this notice and the full citation on the first page. Copyrights for components of this work owned by others than ACM must be honored. Abstracting with credit is permitted. To copy otherwise, or republish, to post on servers or to redistribute to lists, requires prior specific permission and/or a fee. Request permissions from permissions@acm.org.

(C) 2021 Association for Computing Machinery.

2573-9522/2021/07-ART20 \$15.00

https://doi.org/10.1145/3442680 


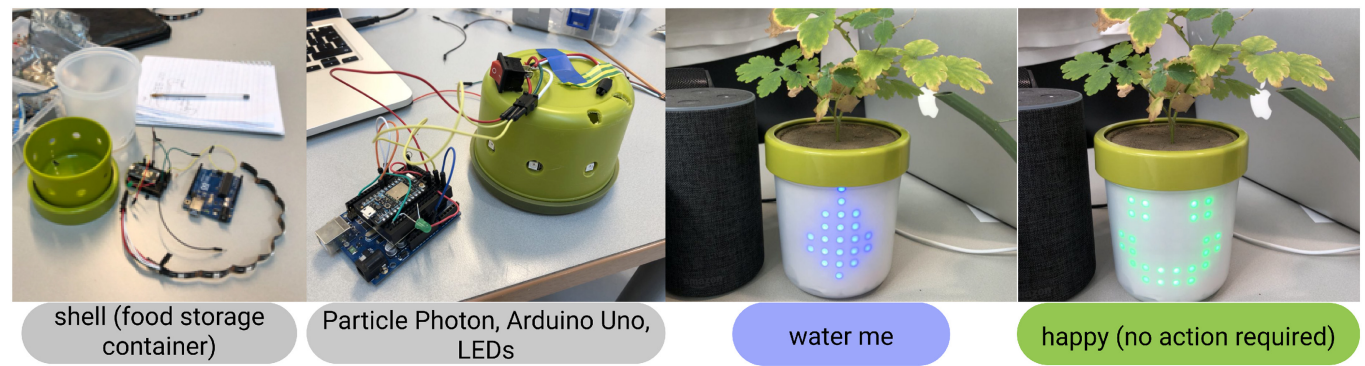

Fig. 1. Design of a plant robot to remind young adults with depression of their behavioral activation therapy tasks at home. From left: Shell, electronics, notification example, and task completed example.

and Prevention [55], almost 30\% of students across the United States have admitted to feeling "sad or hopeless," with $13 \%$ to $20 \%$ having seriously considered suicide.

The need for effective depression management methods is growing. Epidemiological data indicate that problems related to mental health are increasing in both their prevalence and severity [32]. The one-year prevalence of depression was around 4\% to 5\% among UK adolescents from 1976 to 2006 [36] and about 10\% among U.S. young adults in 2014 [46]. Depression has many serious consequences, such as social isolation and poor professional or academic performance. Those with depression can be discouraged from seeking help due to social stigma associated with it $[4,12]$.

One prevalent form of therapy for depression is Behavioral Activation (BA) therapy. It is a psychological treatment that has gained increased attention in recent years. BA therapy conceptualizes depression as an illness that is a direct result of a lack of positive reinforcement, and attempts to counter negative patterns of avoidance, withdrawal, and inactivity by having depressed individuals re-engage in simple activities in their daily life. These activities are designed to be a source of positive reinforcement, which can serve as a natural antidepressant [35].

Meta-analyses on outcomes of BA therapy have shown it to be an effective treatment for depression $[20,21]$. Additionally, BA is less intensive, more cost effective, simpler, and at least equally effective as Cognitive Behavioral Therapy (CBT) (cf. [5, 53]). CBT focuses on addressing current problems by developing self-awareness by monitoring and shifting negative thoughts into positive ones [44]. As BA therapy has advantages over CBT, it makes it a promising form of treatment $[34,53]$. It is the most widely used intervention method for the treatment of depression [6].

Some challenges with traditional BA for young adults is that they may have difficulty following the recommendations of their therapist at home. Young adults also face challenges with obtaining treatment for depression. They may be particularly affected by social stigma about depression, which can make young people reluctant to seek help [4, 12]. Social stigma associated with depression as an illness can cause young adults to drop out of depression-related therapy at dropout rates ranging from $16 \%$ to $72 \%$ [19]. What makes treatment even more difficult is the fact that most therapy models for adolescents are based on treatments meant for adults, meaning that they do not often translate well to adolescents due to their developmental immaturity [7]. As such, interactions with adolescents in depression therapy must be more proactive and directive. Additionally, maintaining a positive attitude and expressing confidence in the therapy progress is essential [7].

The focus of the current work is on adolescents and young adults with depression who undergo BA therapy. Our goal is to develop and evaluate the usability of a prototype that represents the final concept (Figure 1), which can be used to motivate young people to complete the tasks resulting from their therapy on a day-to-day basis. The final concept needs to be able to function as a motivational tool that reminds depressed adolescents to do the tasks set by their therapists. 
Table 1. Assistive Technology for People with Depression

\begin{tabular}{llll}
\hline Name & User (sorted by group) & Therapy & Type \\
\hline Moodlytics & 13 years \& up & Mood tracking & Self-help \\
What'sMyM3 & Adolescents, adults & Screening & Self-help \\
Behavioral Apptivation & Young adults & BA & Therapist monitored \\
WoeBot & Young adults, adults & CBT & Self-help \\
Moodivate & Adults & BA & Self-help \\
MUBS & Adults & BA & Self-help \\
Depression CBT Self-Help Guide & All & CBT & Self-help \\
Breathe2Relax & Military & Anxiety & Self-help \\
T2 Mood Tracker & Military & Mood tracking & Self-help \\
\hline
\end{tabular}

Additionally, it needs to address the issues of being engaging, of not requiring too much effort on the side of the depressed person, and of accompanying the person throughout the depression therapy sessions.

Our contributions are (1) a novel microcontroller-based prototype of a plant robot as a home technology aid for BA therapy, (2) a user-centered design process with two iterations of design and usability evaluation, and (3) an online video-based panel study of young adults' predicted usage and impressions of the system.

\section{BACKGROUND}

\subsection{Assistive Technology for Depression}

Using technology to augment depression therapy is a growing field due to being less intensive, less expensive, and able to treat using empirically validated methods [49]. Most past technologies for depression involve mobile/web applications with different use cases, such as helping track and present analytics about a user's mood, offering articles on depression and therapy, using data collection to evaluate if professional intervention is necessary, or attempting to counter negative emotions by using meditation (Table 1). Here we describe several such systems for adolescents/young adults, general adults, and the military; we also briefly review robots to assist those with depression.

2.1.1 Mobile/Web Applications for Adolescents and Young Adults. Mobile apps, websites, and a chatbot have been developed for adolescents and young adults with depression. The therapy purposes range from mood tracking, screening, BA, and CBT.

Moodlytics [47] developed a mobile app that tracks moods in extensive detail (reasons for mood, people responsible for moods, etc.). It allows users to attach notes, tags, images, and so forth to each mood. The data gathered from the user's mood is then presented in a visually appealing manner to keep the user engaged. The app also performs a "root cause analysis" and helps users identify positive/negative influences in their lives. It also lets users set personal goals and tracks the progress of these goals. It claims to be focused on various groups including teenagers or students (ages 13 to 20), professional adults (ages 20 to 50), retired persons (age 50+), and others.

What'sMyM3 [33] is a website that screens for depression, anxiety, or other mental health conditions using a test and provides screening results and recommendations. It also provides separate screens for adolescents.

Behavioral Apptivation [16] created a mobile phone app that is used for the implementation of BA therapy. Therapists can refer their patients to use the device. It makes therapy more accessible by having therapy material available on the patient's device and helps with planning and 
preparation of therapy easier for the therapist, who can review the patient's daily monitoring data remotely via a therapist website and send positive feedback for progress made. It provides task scheduling, reminder functionality, and goal tracking. A study with 10 therapist trainees and a non-clinical sample of 11 adults averaging 25 years old found it reduced depression by $43 \%$.

WoeBot [22] is a conversational-agent-based therapy companion that engages users in chat conversations. It provides features such as mood tracking, mood analysis, CBT techniques, and conversational companionship. It focuses on bettering a user's mood by engaging the user in conversation and asking questions to facilitate the users' own insights into their mood, and provides positive reinforcement feedback, jokes, and other psychological techniques. WoeBot is one of an emerging line of conversation bots that draw from psychology to motivate the activity design component of HRI (e.g., SwitchBot [69] uses psychology-inspired strategies to help workers detach and reattach from work). A randomized controlled trial with 70 adults aged 18 to 28 years (average age 22 years) found that the chatbot reduced depressive symptoms significantly more than a content control. It was original designed for college-aged adults but is available for anyone.

2.1.2 Mobile/Web Applications for the General Adult Population. Two notable apps have been developed for BA therapy targeting the general adult population. We also note one app for CBT, as well as apps for military personnel.

Moodivate [18] is a self-help sister app of Behavioural Apptivation. Unlike Behavioural Apptivation, which needs patients to be enrolled with a therapist, Moodivate attempts to deliver BA therapy through a self-help framework. As such, this is ideal for individuals who feel down/depressed but are not interested in enrolling for therapy. A randomized trial [17] with 52 adults (averaging 44 years old) found that $70 \%$ were using the app 1 month after deployment and had significantly lower depression at the end of the trial.

MUBS [54] is a mobile app for adults to follow BA activities. It features an activity scheduler and completion tracker, as well as personalized activity recommendations based on validated lists of activity content.

Depression CBT Self-Help Guide [24], developed by ExcelAtLife, is a self-help app with screening tests, a cognitive thought diary (similar to a mood journal), audio clips for relaxation and meditation, articles, and exercises based on CBT.

Breathe2Relax [1] is an app developed by the U.S. Defense Health Agency for the military community. It helps users stabilize their mood and reduce anxiety through breathing exercises that utilize "diaphragm breathing" taught with videos. Its claimed benefits include low stress, reduced anxiety, increased energy, and mood stabilization.

T2 Mood Tracker [11] is a mobile app for military personnel to track symptoms of depression, PTSD, and other conditions. Eight soldiers used the app for 10 different days and found it useful and beneficial. Military health providers were enthusiastic about its use.

2.1.3 Assistive Robots. Some concerns with existing app-based technologies for depression include that they use text, as depressed individuals tend to have problems with reading too much text [30]. Cell phone apps can also be a problem for young people, since excessive cell phone use is correlated with depression, problems with self-control, cell phone dependence, and poor school performance among the young $[28,52,58]$. Unsurprisingly, some researchers have explored assistive robots for persons with depression. This body of HRI research primarily looks at robots for older adults (e.g., [37, 56]; cf. [13]).

Paro [68] is a touch- and voice-sensitive seal robot for adults with dementia. Sixty older adults (ages 62 to 95) who participated in sessions where they petted, spoke to, and sang for the robot had a decline in depression symptoms, whereas a control group did not have a decline [37]. 
Past participatory design research [56] showed care staff and depressed older adults (ages 58 to 71) videos and/or live demos of seven robots (Paro, Care-O-Bot, Giraff Plus, Papero, Roomba, Mugbot, and Keepon). Participants co-designed novel concepts such as a Paro robot that could issue reminders and identified values such as low maintenance, social companionship, and healthrelated functionality for future socially assistive robots.

Although not developed specifically for depression symptoms, the humanoid robot Nao [26] was used to assist with depression symptoms of children aged 6 to 10 years diagnosed with cancer [2]. Five children who participated in sessions where a robot spoke in the character of a "baby robot" had significantly lower depression and anxiety after the sessions, whereas a control group did not have a significant change.

Based on this past work, we note that assistive robots designed specifically to assist young adults with depression symptoms are still a relatively unexplored application area.

\subsection{Nature Therapy, Gardening, and Plant Robots for Depression}

Nature environments can have a restorative effect on people [60]. For example, Korpela et al. [40] found that exposing people with depression to nature increased how restored they felt and decreased depression. Browning et al. [10] found that undergraduates felt more restored and had more positive mood after exposure to either plants and trees outdoors compared to sitting indoors.

Gardening (i.e., the act of watering and taking care of plants) can have additional benefits. Past work [25] found that 12 weeks of gardening activities decreased depression in adults aged 25 to 64 years. Although most gardening research is targeted to older adults (e.g., [66]), past work suggests that gardening could have benefits for younger persons [23] and may work with small indoor plants as well [65]. Thus, more work is needed on how plant types affect different populations [38].

A growing number of researchers are exploring the intersection of plants with robotics, particularly as indoor plants can reduce stress or pain and increase attention capacity or aesthetic appeal (cf. [9, 27, 29, 51]). Plant robots have been used for entertainment performance [8, 41, 48], information visualization for recycling [31], home gardening [14], ${ }^{1}$ electronics [59, 63], agriculture $[42,61]$, and companions $[3,62,64] .{ }^{2}$ However, we are unaware of explorations into plant robots that are focused on helping manage depression.

\section{THE PLANTBOT}

To create the best version of products for people with depression, the user is of utmost importance to the design process [57]. We are therefore guided by a user-centered design process. The key factor within this process is that the user is the most important focus within the entire design process [67], from understanding the user to designing and evaluating the prototype.

The current work presents the first stage of development and testing, which is done with a general group of young adults and with online Mechanical Turk workers who have had depression but may not be clinically depressed at the time of the study. As an initial usability evaluation, we presented the system to young adults who provided feedback on functionality and design, medical specialists who had a deep understanding of young adults with depression, and crowdsourced workers with some experience with depression. In later stages of development, testing with end users in BA therapy is needed to validate the effectiveness of the system for clinical practice.

\footnotetext{
${ }_{1}^{1}$ POT: https://www.parrot.com/us/connected-garden/parrot-pot.

${ }^{2}$ Tessa: https://www.tinybots.nl.
} 


\subsection{Therapist Interview to Understand Context}

We conducted an interview with a behavioral therapist and researcher in the Department of Psychology at the University of Twente to gain a practical understanding of depression, its symptoms. and its treatment. According to the therapist, two types of tasks are usually assigned as part of BA therapy:

(1) Pleasurable tasks: These tasks are primarily intended to be pleasurable to patients and hence are usually highly personal and different for each individual. Tasks of this type may include listening to music, watching a movie, meditating, taking a walk, talking to a loved one, and other tasks that the individual can enjoy. This makes BA a very personal and customizable treatment.

(2) Useful tasks: These tasks are usually performed to gain a sense of accomplishment and achievement. This can range from small, seemingly less important tasks (e.g., cleaning their room) to more important, harder tasks (e.g., doing homework). These tasks are more general and hence can be blanket assigned to different patients.

Assigning these tasks is a key part of depression therapy. However, once the tasks have been assigned, it is up to the patient to complete them, and here lies one of the main issues in BA therapy. One of the key reasons where adolescents face problems in therapy, according to the therapist, is sustaining the motivation required to complete these tasks on a daily basis. This lack of motivation to partake in pleasurable/useful activities can seriously affect the BA therapy and can be a serious impediment to curbing depression, especially in adolescents. Addressing this lack of motivation is necessary in the treatment of depression using BA and is also the conclusion from our literature search (Section 1).

We asked three depression therapists at the University of Twente to complete a questionnaire that explored potential robot designs. The questionnaire first showed static images of four robots: Softbank's Pepper, Sen.se's Mothersense, Amazon's Echo, and Omate's TrueSmart/Yumi. This was followed by videos of each robot and questions about the tasks the robot should do. For appearance, the experts did not like the Mothersense robot and preferred the Omate Truesmart and the Pepper. However, when asked which robot they would like most for reminders, motivational tasks, and to put on display, one of the therapists changed their answer from Pepper to Mothersense. The reasoning was that the robot had a very friendly face and a very nice smile, which they found peasant. The other therapist also changed their answer to Mothersense after movement had been shown. One therapist preferred the Omate Truesmart from the start and did not change their answer. For tasks, therapists gave high priority to tasks that could help within therapy (e.g., listening to music, going for a walk, reminding of tasks) and not to Alexa's existing tasks (e.g., ordering food and recommending movies).

From the survey conducted with the experts, we can conclude that a friendly face could really help in the motivation part of the robot: both the Mothersense and the Omate Truesmart have a friendly face. In addition, the therapy-related tasks we discussed previously with the therapist in the interview should be included within the prototype and the user testing, whereas generic tasks that Alexa normally does could be included but should not be tested in the current work.

\subsection{Design of First Prototype}

The goal of the design stage was to develop a prototype suitable for a usability test in which the interactions of users with the plant robot could be evaluated. Since the prototype needs to be capable of presenting the interactions with the plant robot as realistically as possible, we used Wizard of $\mathrm{Oz}$ to achieve a high level of system responsiveness. We prototyped three tasks: plant 


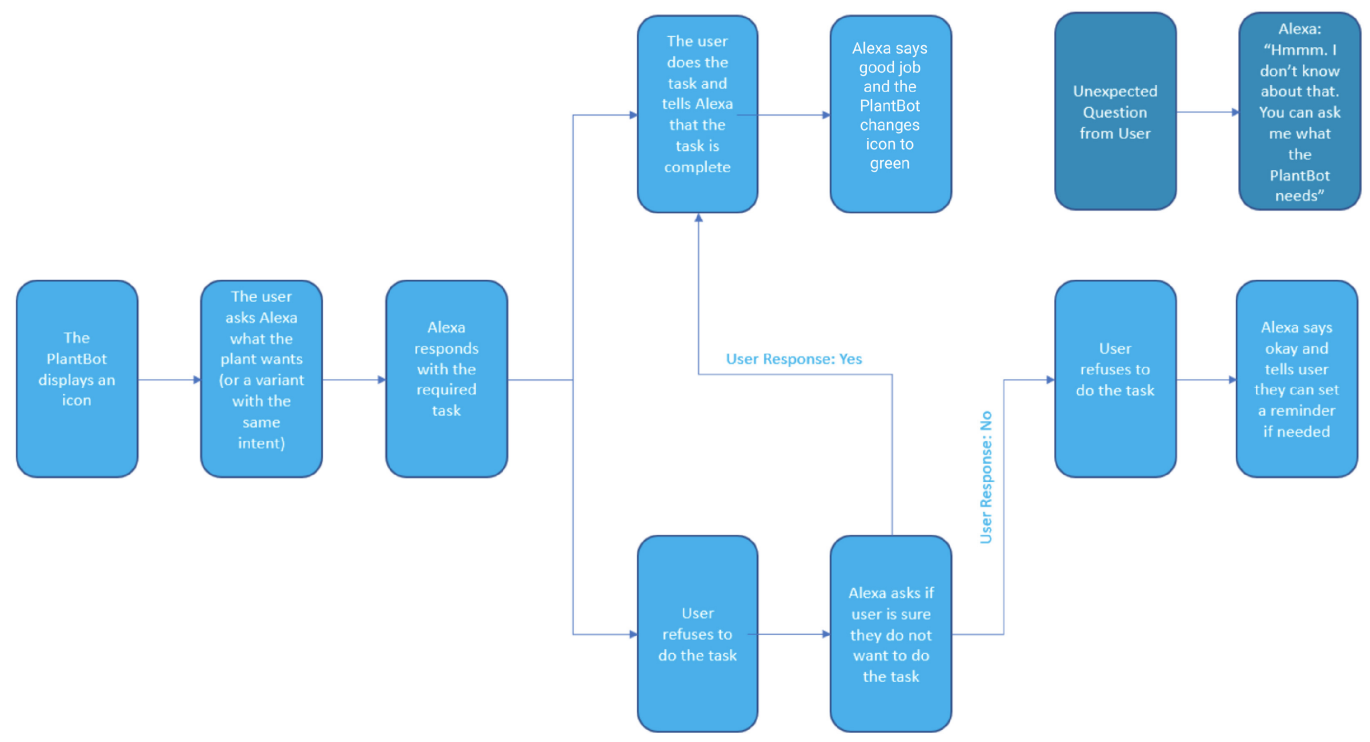

Fig. 2. Dialogue flow among the user, Alexa, and the plant robot.

needed to be watered by the participant (orange light), plant had received sufficient water (green light), and plant robot had a notification for the user with an assignment that had to be carried out (blue light).

If the plant needs water, its lights will always turn orange. This task will trump any other task. This means that blue lights could change to orange when the humidity level drops below the threshold. If the plant has been taken care of, the lights will turn blue to the unfinished task. There can only be one blue task that needs to be performed at a time. When the lights on the plant turn blue, the user must ask the Amazon Alexa what the plant robot wants. The user must always first activate the Amazon Alexa by saying "Hey, Alexa," followed by "what does the plant robot want?" If a blue light task has been performed, the lights will always turn green. There will be a break of at least 30 minutes before next task will be asked. The full dialogue path is shown in Figure 2.

The plant robot is an added module to the Amazon Alexa. The Amazon Alexa can still perform all of its normal tasks and can also be connected to other modules. The plant robot will have an LED strip in the top border of the pot. The LED light has three different color stages as indicated earlier, namely green, orange, and blue.

\subsection{Construction of the First Prototype}

To save time on the fabrication of the prototype's exterior and make it more aesthetically pleasing, the prototype's exterior is a plastic, stackable food storage cup. It consists of an inner and an outer cup, which fit into each other. The inner cup also has a rubber lid for closing. The electronics to display the behavior of the prototype were integrated into the inner cup (Figure 3).

We used two rapid-prototyping boards: a Particle Photon and an Arduino Uno. The Particle Photon prototyping board is a very small board with limited input and output ports. Its usefulness is that it can make a Wifi connection to the Internet and Particle Cloud. Variables and their values stored in the board's memory are shared with the Particle Cloud. Most importantly, however, is that the cloud functionalities allow for these values to be edited externally from the board itself. This enabled the development of a controller to manage the interaction states of the prototype. The Arduino Uno is a larger prototyping board that is capable of providing more power to other 


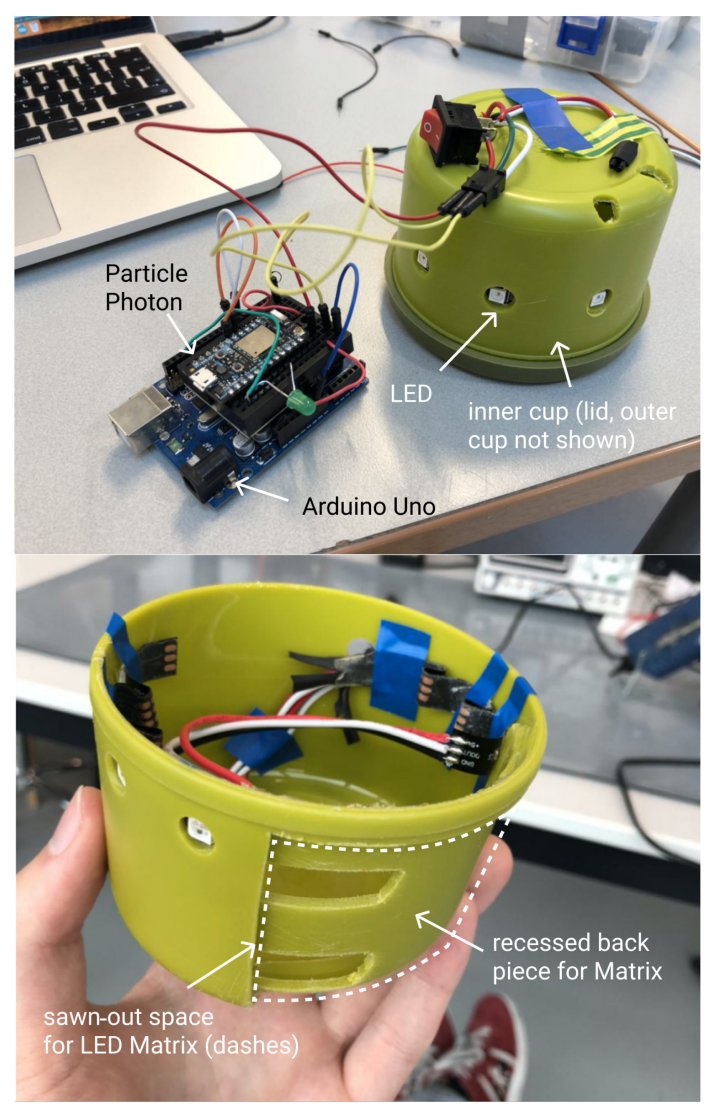

Fig. 3. Electronics within the Tupperware body of the robot.

hardware connected to it compared to the Particle Photon. Within the prototype, its the main purpose is to provide the power and data input to a LED strip that is coiled around the prototype's body. The Arduino receives data on the prototype's state from the Particle Photon board. Based on this input, data that manipulate the LED's color and brightness will be sent to the LED strip.

Both of the prototyping boards were placed at the bottom of the outer cup. The wires to power and control the LED strip were routed through the inner cup in which holes were drilled so the individual LEDs could shine through.

To prevent the participants from seeing the electronic components on the inside of the prototype, a thin layer of cardboard was wrapped around the cup. This cardboard also diffused the light from the LEDs and made them appear more smooth.

To provide the boards with power, two small holes were drilled at the bottom of the outer cup so that the power cables could go through and reach the boards.

Finally, a fake plastic plant was attached to the prototype by routing through a hole in the rubber lid on top of the inner. This fake plant made the prototype appear more as intended in a final version of the plant robot.

To control the prototype during the user test, a web-based control interface was developed. The website displayed the current state of the prototype and incorporated switches to change the current state. By toggling a switch, a command would be fired to the Particle Cloud to update the 
status; the Particle Photon would then register that the state of the prototype has been changed and in turn send this information to the Arduino board.

For our first prototype, Alexa's responses were typed into the Alexa developer console by a wizard and played through a Bluetooth speaker. This gave the impression that Alexa did the talking herself, although she was actually turned off since automated speech was not reliable enough.

\subsection{Pilot Test with the First Prototype}

We ran a pilot test with 30 participants selected from the Design Lab at the University of Twente; we did not explicitly recruit people with depression (i.e., depression screening questions were not used). Each participant was told that the lights of the plant robot indicated its current state and that Alexa is a home automation product that can be activated by saying "Hey, Alexa" (in the pilot test only, Alexa would state the task if the participant did not do anything for 30 seconds). A wizard operated the robot's lights and dialogue (Figure 2) for each of three tasks. The main points of improvement were that participants did not understand that the blue lights indicated watering the plant (the meanings of other lights were clear) and the responses from Alexa were delivered too slow through the Alexa Developer Console. An additional interesting finding was that some participants performed tasks simply because the plant wanted it.

\subsection{Design of the Second Prototype}

The second prototype explicitly defined the interaction protocol: the Plantbot makes a sound and changes its screen when a task arrives but then does not make more sounds until the user initiates a dialogue with Alexa. This is because conversing with Alexa guarantees that the user is present and because repeated sounds decrease the system's inconspicuousness and may draw too much attention to the device. Based on the pilot test, we supported this interaction by adding a screen to display icons and included new states so that the PlantBot could respond to a series of declined or completed tasks. We also included a speaker so that the PlantBot could make sounds to accompany the visual feedback and implemented faster spoken responses. The states were as follows:

- Water-droplet: This icon is used to indicate that the PlantBot needs water. It is a blue droplet to refer to the color and shape often associated with water. When the display transitions to this icon, a sound of water droplets gliding down and splashing on a surface is played.

- Happy Emoticon: This is displayed when a task is performed correctly or the plant has received water upon request. It communicates that the PlantBot is satisfied by using a green smiling emoticon. The accompanying sound when entering this state is an ascending arpeggio in a major scale (a quick and happy succession of notes).

- Question mark: This icon appears when the PlantBot has an update for its user, such as a message containing a reminder or nudge to perform a certain task. The sound that plays with the icon was inspired by smartphone notification sounds: we adapted it slightly however so that no confusion will occur between the smartphone and PlantBot notifications.

- Sad Emoticon: When the user refuses to fulfill a task, the PlantBot will first try to nudge them into performing the task by explaining why it would be beneficial to do it. Should the user still refuse, the screen will display a sad light blue emoticon. Contrary to the sounds displayed with the Happy Emoticon, a descending minor arpeggio will be played.

- Heart Icon: As an alternative to the Happy Emoticon, the heart icon (red color) is displayed on special occasions or when the user does multiple tasks correctly in relatively quick succession. For this icon, the sound is currently identical to the sound that is played with the Happy Emoticon. 
For our second prototype, Alexa's responses were pre-recorded using the Alexa skill set and loaded into a web-based control interface for faster interaction.

\subsection{Construction of the Second Prototype}

To make the screen fit inside the prototype, various changes had to be made to the inner cup of the prototype. A major section of the inner cup was sawn out in such a way that an LED Matrix would fit in its place. This sawn-out section then formed a recessed backing for the LED Matrix. An LED strip was split into two pieces placed on either side of the LED Matrix so that the Matrix and strips could produce a single, equally spaced ring of LEDs along the top of the cup. We used a flexible (i.e., able to bend along the cup's curve) Adafruit $8 \times 8$ NeoPixel RGB LED Matrix. ${ }^{3}$

Finally, to make the PlantBot prototype more realistic, the lid of the original cup was modified to make it look more like an actual plant pot. This was done by gluing sand to the rubber lid using wood glue (because this glue becomes transparent after drying and does not affect rubber like most glues do). Furthermore, an actual plant was pinned through the lid to make it appear as if it were actually planted inside the earth of the plant pot.

\subsection{Usability Study of the Second Prototype}

3.7.1 Participants. We recruited 20 participants from the Design Lab at the University of Twente; participants were from the general student population, as we did not include depression screening questions. Participants from the pilot study were excluded. We also showed the system to a domain expert, a therapist-researcher at the University of Twente.

3.7.2 Procedure. The basic procedure of the user test is the same as with the first user test, with a few changes. After participants were introduced to the robot and Alexa, we asked participants to "Please ask Alexa if she can tell a joke" as a practice step. We then had the participant experience three task requests: watering the plant, cleaning the desk, and selecting a song for Alexa to play. Each request began with the PlantBot's LEDs turning blue, followed by the dialogue with Alexa. At the conclusion of the study, we asked participants about interaction initiation ("Did Alexa initiate or did you initiate?"; "Did you understand you had to initiate the conversation?"), voice design ("Do you think Alexa's voice is motivational?"), visual design ("Which icons did you see?"; "Did you understand the icons?"; "Did you feel compelled to [water the plant/clean the desk/listen to music]?") and suggestions ("Would you change or add anything to the interaction with the plant?").

3.7.3 Analysis. We first calculated the proportion of participants who mentioned they understood each component of the system. We also used a consensus method for qualitative analysis of responses from the usability test: the research team met to discuss every participant's responses, identify notable insights from the data, and come to an agreement on the most important points (rather than coding individually). We selected this method because it supported group discussion of the entire dataset and consensus-based selection of its notable insights ("team research" [15]), but we could not assess rater agreement as can be done with more formal methods.

3.7.4 Results. For the task of watering the plant, 15 participants initiated the conversation with Alexa, whereas 5 participants waited until Alexa asked them to water the plant. All but one participant watered the plant. The one participant who did not was confused about the fact that there was no water in the watering can. This participant was not the only one confused about the reallooking plant, which seemed to stand in real dirt. In total, 7 participants were confused about this

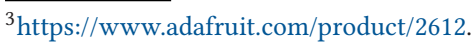


mismatch of appearance and lack of water but pretended to water anyway after Alexa prompted a second time, saying that they should use the watering can.

After the question mark ("?") symbol was shown to participants for the clean desk task, 19 out of the 20 total participants actually initiated the conversation. Fourteen participants cleaned the desk when asked to, 4 did it after the second prompt, and 2 participants refused to clean the desk. One of the participants who refused to clean the desk told Alexa he did, effectively cheating the system. Six participants also wondered why the PlantBot would care if their desk was clean, as to them it did not make sense that the plant needed a clean desk.

The third task was listening to music. When the plant prompted the participant again with the question mark, 19 participants responded by initiating the conversation, whereas 1 participant needed a second prompt. All participants selected a song to listen to.

During the user test and subsequent interviews, some general remarks about the prototype were made as well. One participant indicated that if the plant was happy, he was also happy-indicating the a happy plant could motivate him or her to perform certain tasks, which is exactly the goal of the prototype. Another user was confused about the minimalistic water sound the PlantBot made when it prompted for water the first time. To the participant, it seemed like the PlantBot was watering itself. One participant asked for a second joke in the middle of the user test, to which Alexa responded by telling him another one of the five implemented jokes. The participant became very happy and satisfied with this action, after which the test continued along the set path.

Participants also expressed some confusion about the user interface. Some were confused about the question mark icon, indicating that they thought the plant had a question for them instead of a task or message. They argued that it would have been better to use an exclamation mark ("!"). Some reported that the water droplet looked like a diamond rather than a drop, likely due to the low-resolution LED Matrix. Some other remarks regarding the prototype included the feeling that Alexa was ordering them to do something instead of asking or suggesting them to do something. This made some of the participants a little uncomfortable. One participant suggested that it might be good feedback to make the plant wiggle when a certain goal was achieved. Finally, many participants found it strange that Alexa and the PlantBot were two separate entities; they suggested that they should be merged.

The domain expert approved of the design in general but made some suggestions. He questioned whether taking care of a plant would be attractive to young people. Since adolescents are not typically enthusiastic about gardening, the appeal of the device may vary from user to user. However, he also noted that disguising a smart device as a plant makes it stealthy and will not stand out. Due to the stigma associated with depression, having a device that is not obvious as an assistive therapeutic device would be attractive to a lot of potential users.

According to the therapist, the combination of icons and sounds to provide feedback can be seen as rewarding, as the user is given positive feedback for completing a task and encouragement in case a task is not complete. This was seen in the user testing when some users felt elated due to the plant being happy after the completion of a task.

The therapist preferred the current method of the PlantBot initiating the conversation through sounds and icons over having Alexa initiate through voice, because voice may be too intrusive if the device is placed in an open area in the patient's home. Additionally, the therapist suggested that increasing the rewards system could be beneficial in motivating the users. Along with icons and sounds as positive feedback, it could be useful in gamifying the task completion activities through a point system for rewards. He also added that the user could also be free to select their own rewards, which can lead to higher satisfaction on completing tasks.

Overall, the therapist liked the design of the PlantBot and sees value in developing the idea further. 
3.7.5 Conclusions. From the second user test, we can conclude that the interaction with both Alexa and the PlantBot was greatly improved with the second prototype. However, there remains a lot of room for improvement regarding user feedback and the overall workings of PlantBot. Feedback can be improved through clearer and more diverse icons and sounds. Alexa's conversation flow has a lot of room for expansion, as does the amount and types of tasks available. Nevertheless, the user test seemed to show that the overall concept and idea behind PlantBot could be helpfulin particular, the interaction of displaying lights to signal to the user to speak with Alexa about a task.

\section{ONLINE PANEL STUDY}

The usability study assessed the prototype with a general pool of young adults; we next wanted to assess its usability with young adults with knowledge of depression. We therefore looked at how young adults who have had depression perceive our plant robot prototype that issues reminders for BA therapy tasks in an online, video-based study. A systematic review of 139 papers on depression technology found that $30 \%$ evaluated their systems using typical user studies, whereas only 16 used clinical evaluation, defined as evaluation with persons with depression, usually in a clinical setting [57]. However, that review also noted that it is difficult to acquire resources to conduct a clinical trial and questioned the appropriateness of clinical trials for early-stage, non-participatory development of new technologies, due to the effort it places on clinical participants. In light of these competing considerations, we propose a method that alleviates the additional effort of clinical research on both the researcher and the participants: an online panel study. We define this as a two-step method whereby a population of crowdsourced workers much larger than the desired number of participants is asked screening questions on a variety of metrics, including, but not exclusively, the target metric (e.g., past depression); only participants matching the criteria are then contacted about the survey in question. This two-step method gives researchers more confidence that their participants have been previously diagnosed with depression than if the researchers create a generic task available to all crowdsourced workers asking for people with past depression, which will surely result in people falsely reporting that they have had depression in order to participate in the task. With this two-step method, crowdsourced workers do not know recruitment criteria beforehand, so they are less likely to falsely report it. This method also has the advantage of being very quick (i.e., less than 10 minutes) for participants to complete, at a time when they are presumably going to work on crowdsourced tasks anyway.

\subsection{Method and Materials}

4.1.1 Participants. We used CloudResearch's Prime Panels [43], which periodically samples millions of MTurk workers for various demographics, to identify MTurk workers with past depression. Our MTurk task was sent only to workers who had indicated past depression in a prior CloudResearch survey ("Have you ever been diagnosed with the following conditions? Please select all that apply"; in turn, they selected "Depression").

We recruited 30 U.S. MTurk panel workers (19 female, 11 male) ages 19 to 24 years $(M=21.9, S D=$ 1.8) from a subset of more than 7,000 workers who had reported a past diagnosis of depression. Participants were paid $\$ 0.48$. Survey duration was approximately 4 to 7 minutes.

Participants' therapy experience was as follows: 1 participated in both CBT and BA, 16 participated in CBT only, 1 participated in BA only, and 12 participated in neither CBT nor BA. Participants' therapy knowledge was as follows: CBT $(M=3.0, S D=1.3$, on a 5-point Likert scale from 1 being "Not at all knowledgable" to 5 being "Very knowledgable") and BA $(M=1.8, S D=1.0)$. All answered "Yes" to having ever been diagnosed with depression. We did not ask about participants' current diagnoses. 
4.1.2 Study Design. A two-condition, repeated measures study (judgment target: self vs peer) was conducted online. The paired dependent variables were anticipated request-following behavior (i.e., would the target follow the robot's request) and anticipated feeling toward the robot. Two additional unpaired measures, which had one "self" condition only (i.e., no peer condition), were suggestions for improvement and opinion of the plant component.

We included these conditions as an attempt to arrive at more authentic responses, given that past work in robotics (cf., [45]) found that some elderly participants report a robot as appropriate for others but not themselves. It also served as an exploration of whether young adults may also report differences in the suitability of technology for themselves versus their peers.

4.1.3 Procedure. The survey was structured as an introduction section, a video, and a questionnaire section. After an information and consent page, participants were asked to "Please imagine you are at home and you recently received the home technology shown in the following short video. Please imagine yourself (instead of the actor) experiencing the technology." They then viewed Video 1 (see the supplemental material) featuring an actor using the system. The actor viewed each icon and sound from the plantbot, then performed the correct task. After the video, participants were asked "[Would you/Do you think people with depression would] do the requests made by the home technology shown in the video? Please explain briefly." They also were asked "[How do you/How do you think people with depression would] feel about the home technology shown in the video? Please explain briefly." These two questions were asked to assess predicted behavior and emotions, respectively. (Both questions in brackets were asked for each person.) We then asked "Do you have any suggestions for the home technology shown in the video?" and "What do you think about designing the home technology to look like a plant?" All procedures were approved by the ethics board at the University of Twente.

4.1.4 Analysis. We coded self/peer request-following behavior as Yes, Maybe, or No, as well as the presence of the following reasons: companion, task simple, helpful, interesting, unhelpful, mood/symptom, and weird. Reasons were based on inductive open coding of main points in the responses, followed by grouping open codes to a final list and then recoding all responses for each code in the list. Sometimes open coding resulted in opposite reasons (e.g., helpful, unhelpful), which was kept. For self/peer emotion, the researcher first coded it as Positive, Neutral, or Negative, then coded responses for the presence of the following feelings (in the same manner as earlier): enjoyable, helpful, motivating, interesting, annoying, weird, indirect, unhelpful, and other. Responses to the plant question were coded as Liked or Not Liked and then into categories using the open coding method presented earlier. Participants' suggestions were also coded using open coding. Self request-following behavior had four missing values from people who described the behavior in the video rather than whether they would follow the system's requests; these values were omitted.

\subsection{Results}

4.2.1 Plant Component Is Alive, Cared for, and Discreet. A total of 28 out of 30 (93\%) participants liked the plant component of the technology. One main reason was that a plant appears alive (Figure 4). One participant wrote "plants and nature in general tend to help elevate the mood of most people, and having it look like a lifeform rather than a static piece of machinery is quite a good idea." Furthermore, some participants suggested to actually use a real plant: "I think it should come as a pot with a little small plant you can grow in it." On a similar note, participants also wrote that a plant is positive (e.g., "I think it helps ... a lot of people have positive associations and feelings for them") and can be cared for (e.g., "having a plant to take care of can help people feel responsible and more happy"). Another key reason participants mentioned is that a plant is 


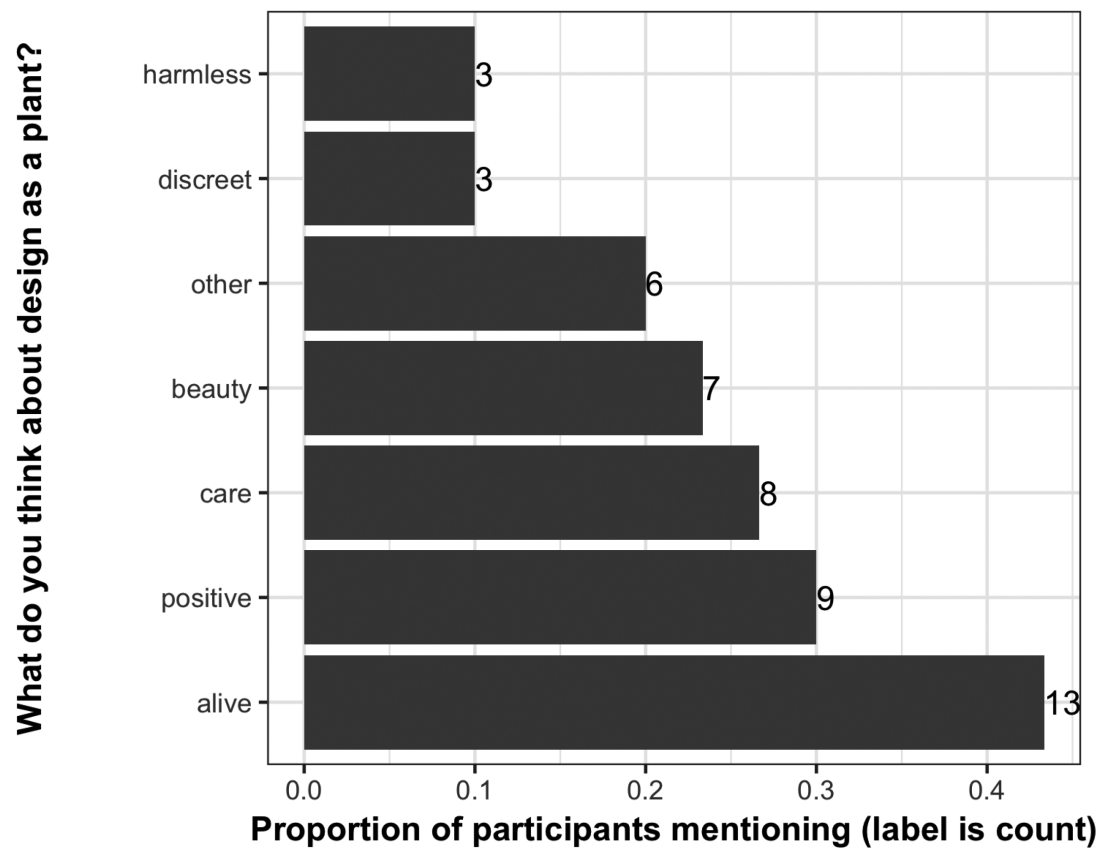

Fig. 4. Proportion of participants mentioning each opinion (code) about the plant component of the robot.

discreet. For example, one person wrote "designing it to look like a plant was smart as it fits in more with people's decorating scheme and can be easier to disguise if they didn't want anyone to know that they had it."

4.2.2 Lack of Appropriate Tasks. Several participants said that the tasks we prototyped were not valuable to their specific lifestyles. For example, three participants said the music task was useless to them because they do not like listening to music ('I wouldn't play the music bc I don't like listening to music while I do work"), and one said they would already be listening to music, so they would not need the plant to remind them ("I would probably have music playing already"). This is coded as "unhelpfulness" in Figure 5. This suggests that the specific tasks shown in the video had a substantial effect on some participants' answers about their own anticipated usage of the device. However, such comments were not present when participants answered about how other young people with past depression would follow the recommendations of the plant.

Participants gave some suggestions to fix the limited tasks. Three participants suggested to "expand the range of commands." Some gave further suggestions of specific tasks, such as a "calendar would be helpful too (reminder that there's a party to go to)." Alternatively, other participants wanted to create the tasks themselves ("I'd try it out, especially if I could program the types of suggestions that the plant gives"; "I would want the suggestions to be programmable and have some on a timer").

4.2.3 Merging Alexa and PlantBot. Several participants strongly suggested to have only one device. These participants either wanted the plant robot removed and merged into Alexa ("should be integrated into Alexa instead of as a separate device. By itself, I think it is useless") or they wanted Alexa to be removed ("maybe have the plant talk in stead of alexa"). For these participants, it did not seem that the benefit of having both a general-purpose Alexa and a companion device in a plant shape was worth the cost or hassle of having two devices. 


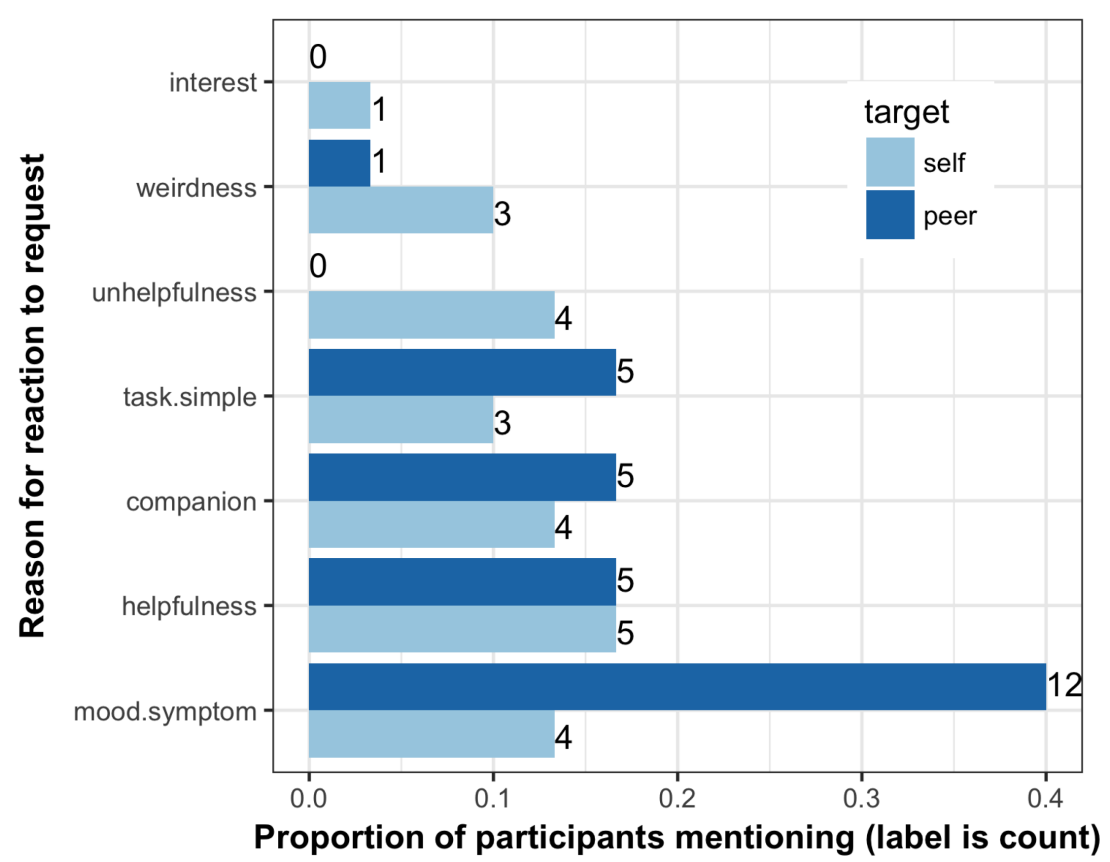

Fig. 5. Effect of peer on proportion of participants mentioning each reason for their own or their peers' reaction to the robot's request.

4.2.4 Anticipated Request-Following. Overall, 13 (50\%) out of 26 responses reported that they would probably follow the requests of the system (4 responses were omitted for describing the video). The most mentioned reasons for this, based on the coding, were as follows: they thought the task was valuable (five mentions; e.g., "Yes, because it would give me a sense of accomplishment"), they wanted to keep the plant happy (three mentions; e.g., "Yeah, I think that I would. It seemed like a nice little buddy"), and that the task was simple (two mentions; e.g., "yeah i would probably do the tasks it asked me to do because they are fairly simple") (Figure 5).

Nine participants (35\%) who qualified their answers with contingencies (e.g., "Some of the more simple tasks I would maybe do" and "Only if it directly helps the plant") were coded as maybe following the requests.

Four participants (15\%) were coded as anticipating not following the requests. Three of these participants mentioned task unsuitability as the reason (e.g., "I would probably not play music if I was working" or "This technology was pretty annoying with tasks that weren't even that important or meaningful"). The fourth participant mentioned mood/symptoms as the reason: "I probably would not do the requests as I often get so caught up in what I'm doing I end up ignoring my other responsibilities and tasks."

We assessed the effect of peer on participants' anticipated request-following behavior. We conducted a McNemar chi-squared test with continuity correction on a $2 \times 2$ contingency table of anticipated request following of self versus peer judgment target in a sample of 26 participants (Table 2). The effect of self versus peer was not significant, McNemar's $\chi^{2}(1)=1.8, p=0.18$. We found no evidence that young adults with past depression anticipated their request-following as being different from their peers' request-following.

We also tested whether self versus peer judgment affected the distribution of reasons for people's response to the robot's request. Pearson's chi-squared test on the count distributions of 
Table 2. $2 \times 2$ Contingency Table for Anticipated Request-Following Behavior for Participant's Self and Peers

\begin{tabular}{lcc}
\hline & Peer: Maybe or No & Peer: Yes Follow \\
\hline Self: Maybe or No & 11 & 2 \\
Self: Yes Follow & 7 & 6 \\
\hline
\end{tabular}

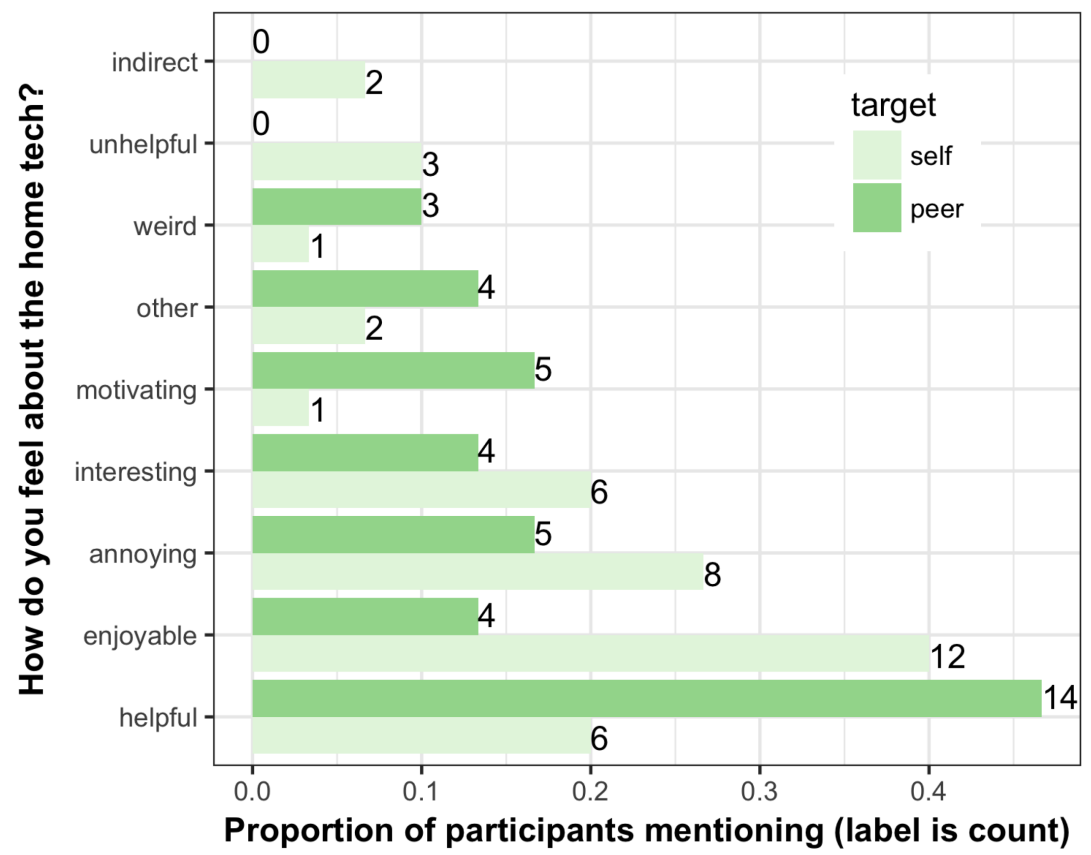

Fig. 6. Effect of peer on the proportion of participants mentioning each reason for their own or their peers' feeling toward the robot.

request-following reasons mentioned for oneself versus one's peers was not significant, $\chi^{2}(6)=$ $10.4, p=0.11$. Reasons for self versus peer behavior in response to robot requests did not differ significantly (Figure 5).

4.2.5 Anticipated Feelings Toward Robot. The most common feeling participants mentioned when speaking about themselves was that the system appeared enjoyable (e.g., "i like it and think it is cute") (Figure 6). Some participants also mentioned that "I would probably be annoyed at times being distracted by it." When asked to anticipate how their peers would feel, the most common emotion mentioned was helpful (e.g., "They could find it mildly helpful").

We tested whether the reported emotions of young adults with past depression differed when asked about their own emotions versus their peer's emotions. Pearson's chi-squared test on the count distributions of emotion categories mentioned for oneself and one's peers was significant, $\chi^{2}(8)=17.6, p=0.02$. Follow-up McNemar chi-squared tests on $2 \times 2$ contingency tables for each emotion category found significant effects of self versus peer rating for helpful emotion (Table 3), McNemar's $\chi^{2}(1)=4.1, p=0.04$, and enjoyable emotion (Table 4), McNemar's $\chi^{2}(1)=6.1, p=$ 0.01 . Young adults with past depression more frequently anticipated themselves as feeling the 
Table 3. $2 \times 2$ Contingency Table for Absence or Presence of Helpful Code in Expected Feeling of Self and Peers Toward Robot

\begin{tabular}{lcc}
\hline & Peer: Not Helpful & Peer: Helpful \\
\hline Self: Not Helpful & 14 & 10 \\
Self: Helpful & 2 & 4 \\
\hline
\end{tabular}

Table 4. $2 \times 2$ Contingency Table for Absence or Presence of Enjoyable Code in Expected Feeling of Self and Peers Toward Robot

\begin{tabular}{lcc}
\hline & Peer: Not Enjoyable & Peer: Enjoyable \\
\hline Self: Not Enjoyable & 18 & 0 \\
Self: Enjoyable & 8 & 4 \\
\hline
\end{tabular}

technology would be enjoyable compared to their peers, and more frequently anticipated their peers as feeling the technology would be helpful compared to themselves.

\section{DISCUSSION}

\subsection{Summary of Results}

We present a new assistive robot with the novel function of helping young persons with depression to follow their BA therapy tasks at home. Compared with past assistive robots or mobile apps, we target an underserved group of young adults undergoing BA therapy, with a new microcontrollerbased, plant-shaped device that is integrated with the Alexa voice assistant. Reviews with a mental health professional provided context and suggested that the system might be helpful. Preliminary usability tests found that the system's verbal dialogue flow and visual icons were understood by young adults. A follow-up online study with 30 young adults with past depression found that $93 \%$ liked the plant component of the device and roughly half thought that the system could be useful.

\subsection{Methodological Implications}

PlantBot encourages more research to help young adults with symptoms of depression. This user group was not fully addressed before in the assistive robot or mobile app literature. Past mobile/web apps and robots to aid with depression have predominantly been designed for general adults or older adults rather than young adults.

The goal of PlantBot is to alleviate users' symptoms of depression; it is therefore critical to involve young adults with depression in the development process. In the current work, we explicitly chose not to involve this target user group because we believe that user tests that are part of a nonparticipatory design process should only occur in the latter phases of product development, so as to avoid testing nascent technology with a vulnerable population too early. We instead selected to run an online panel of young adults with past depression and consultations with mental health experts because of the lower research footprints involved. This methodology helped to determine that the PlantBot component of the system appears ready for usability testing with the user group.

\subsection{Design Implications}

From a design point of view, our results promote new designs of technology shaped as plants rather than machines. We add evidence that plant devices do appear to be promising for youth, since a very high percentage (93\%) of participants liked the plant component of the device. As mentioned by therapists and some participants, the advantages of a plant shape include its inconspicuousness 
and the positive associations people have with plants. Plants and plant-shaped technologies for young persons in other use cases related to health and well-being is a promising area.

A second design finding is that personalization of BA tasks is needed in any app or robot designed for young adults with depression. Our online study received many comments stating that specific tasks we prototyped did not make personal sense for participants and needed to be expanded upon. Expanded task lists are likely more important than plant-related features, such as adding a humidity sensor to monitor the state of a plant. This would help make the system more suitable for long-term engagement. We note that recent BA mobile apps (e.g., [54]) have implemented more than 300 tasks.

A third finding is that some respondents want a stand-alone robot rather than a peripheral device that utilizes an Amazon Echo speaker. Unlike past mobile apps for depression therapy, which use a person's existing mobile phone, many people did not want the plant robot to use a person's existing smart speaker. Their preference implies that a plant robot with a speaker conus and microphone integrated at the bottom of the cup, which connects to the cloud-based Alexa API via a Photon Particle board, is more desirable than a plant robot that signals to the user to speak with the Amazon Echo speaker. The benefits to an integrated device include less cost because the Amazon Alexa does not need to be purchased anymore.

Our results also add evidence that young adults find voice assistant robots usable for assistive purposes. One potential advantage of voice assistant robots is that they are physically present and therefore may be more attention grabbing in a home setting and harder to remove than an app on a mobile phone.

\subsection{Limitations and Future Work}

A methodological limitation of the current work is that the main study was done online. Online survey results may not generalize to in-person behavior with a co-present robot.

Another limitation is that the dialogue was pre-recorded and wizarded for the user tests. A part that needs to be built for the product to be fully functional is the dialogue interaction with Alexa. The dialogue needs to be integrated in the Alexa skills kit, which in turn needs to be able to get updates from the therapist's portal. However, this was beyond the scope of the current work, as the bottleneck in automating this process is the task of updating the dialogue structure automatically depending on the new task assigned. Moreover, a limitation in our analyses was that inter-coder reliability could not be calculated, as we did not use independent coders.

Based on these limitations, we believe an essential system component that should be implemented prior to a field study is the therapist's Internet-based scheduler that sets personalized tasks in the PlantBot. The therapist's scheduler is envisioned as the next step of system development.

\section{CONCLUSION}

We demonstrated how a plant robot can be applied to deliver BA therapy at-home reminders to young adults with depression. We created a prototype of a novel microcontroller-based plant robot that functions with the Alexa conversational assistant. Online usability evaluation of the plant robot with young adults who have had depression found that the plant idea was well liked and that the tested components are ready for further usability testing with the user group.

\section{REFERENCES}

[1] Defense Health Agency. 2017. Breathe2Relax. Retrieved April 1, 2021 from https://health.mil/Reference-Center/FactSheets/2020/10/26/Breathe2Relax-App.

[2] Minoo Alemi, Ali Meghdari, Ashkan Ghanbarzadeh, Leila Jafari Moghadam, and Anooshe Ghanbarzadeh. 2014. Effect of utilizing a humanoid robot as a therapy-assistant in reducing anger, anxiety, and depression. In Proceedings of the 2nd RSI/ISM International Conference on Robotics and Mechatronics (ICRoM'14). IEEE, Los Alamitos, CA, 748-753. 
[3] Leonardo Angelini, Stefania Caparrotta, Omar Abou Khaled, and Elena Mugellini. 2016. EmotiPlant: Human-plant interaction for older adults. In Proceedings of the 10th International Conference on Tangible, Embedded, and Embodied Interaction (TEI'16). ACM, New York, NY, 373-379.

[4] Lisa J. Barney, Kathleen M. Griffiths, Anthony F. Jorm, and Helen Christensen. 2006. Stigma about depression and its impact on help-seeking intentions. Australian \& New Zealand fournal of Psychiatry 40, 1 (2006), 51-54.

[5] Jürgen Barth, Thomas Munder, Heike Gerger, Eveline Nüesch, Sven Trelle, Hansjörg Znoj, Peter Jüni, and Pim Cuijpers. 2016. Comparative efficacy of seven psychotherapeutic interventions for patients with depression: A network meta-analysis. Focus 14, 2 (2016), 229-243.

[6] Eric T. Beeson and Thomas A. Field. 2017. Neurocounseling: A new section of the journal of mental health counseling. Journal of Mental Health Counseling 39, 1 (2017), 71-83.

[7] Keri Bolton Oetzel and David G. Scherer. 2003. Therapeutic engagement with adolescents in psychotherapy.Psychotherapy: Theory, Research, Practice, Training 40, 3 (2003), 215.

[8] Cynthia Breazeal, Andrew Brooks, Jesse Gray, Matt Hancher, Cory Kidd, John McBean, Dan Stiehl, and Joshua Strickon. 2003. Interactive robot theatre. In Proceedings of the 2003 IEEE/RSF International Conference on Intelligent Robots and Systems (IROS'03), Vol. 4. IEEE, Los Alamitos, CA, 3648-3655.

[9] Tina Bringslimark, Terry Hartig, and Grete G. Patil. 2009. The psychological benefits of indoor plants: A critical review of the experimental literature. Fournal of Environmental Psychology 29, 4 (2009), 422-433.

[10] Matthew H. E. M. Browning, Katherine J. Mimnaugh, Carena J. van Riper, Heidemarie K. Laurent, and Steven M. LaValle. 2019. Can simulated nature support mental health? Comparing short, single-doses of 360-degree nature videos in virtual reality with the outdoors. Frontiers in Psychology 10 (2019), 2667.

[11] Nigel E. Bush, Gary Ouellette, and Julie Kinn. 2014. Utility of the T2 Mood Tracker mobile application among army warrior transition unit service members. Military Medicine 179, 12 (2014), 1453-1457.

[12] Alison L. Calear, Kathleen M. Griffiths, and Helen Christensen. 2011. Personal and perceived depression stigma in Australian adolescents: Magnitude and predictors. fournal of Affective Disorders 129, 1-3 (2011), 104-108.

[13] Shu-Chuan Chen, Cindy Jones, and Wendy Moyle. 2018. Social robots for depression in older adults: A systematic review. Journal of Nursing Scholarship 50, 6 (2018), 612-622.

[14] Jaewon Cho, Sanghoo Park, Been Jeon, Byung-Chull Bae, and Jun-Dong Cho. 2015. People's emotional responses to a plant's emotional expression. In Proceedings of the 9th International Conference on Tangible, Embedded, and Embodied Interaction. 545-550.

[15] Flora Cornish, Alex Gillespie, and Tania Zittoun. 2013. Collaborative analysis of qualitative data. In The SAGE Handbook of Qualitative Data Analysis, U. Flick (Ed.). SAGE, 93.

[16] Jennifer Dahne, Jacob Kustanowitz, and C. W. Lejuez. 2018. Development and preliminary feasibility study of a brief behavioral activation mobile application (Behavioral Apptivation) to be used in conjunction with ongoing therapy. Cognitive and Behavioral Practice 25, 1 (2018), 44-56.

[17] Jennifer Dahne, C. W. Lejuez, Vanessa A. Diaz, Marty S. Player, Jacob Kustanowitz, Julia W. Felton, and Matthew J. Carpenter. 2019. Pilot randomized trial of a self-help behavioral activation mobile app for utilization in primary care. Behavior Therapy 50, 4 (2019), 817-827.

[18] Jennifer Dahne, Carl W. Lejuez, Jacob Kustanowitz, Julia W. Felton, Vanessa A. Diaz, Marty S. Player, and Matthew J. Carpenter. 2017. Moodivate: A self-help behavioral activation mobile app for utilization in primary careDevelopment and clinical considerations. International fournal of Psychiatry in Medicine 52, 2 (2017), 160-175.

[19] Anna M. De Haan, Albert E. Boon, Joop T. V. M. de Jong, Machteld Hoeve, and Robert R. J. M. Vermeiren. 2013. A meta-analytic review on treatment dropout in child and adolescent outpatient mental health care. Clinical Psychology Review 33, 5 (2013), 698-711.

[20] David Ekers, David Richards, and Simon Gilbody. 2008. A meta-analysis of randomized trials of behavioural treatment of depression. Psychological Medicine 38, 5 (2008), 611-623.

[21] David Ekers, Lisa Webster, Annemieke Van Straten, Pim Cuijpers, David Richards, and Simon Gilbody. 2014. Behavioural activation for depression; an update of meta-analysis of effectiveness and sub group analysis. PLoS ONE 9 , 6 (2014), e100100.

[22] Kathleen Kara Fitzpatrick, Alison Darcy, and Molly Vierhile. 2017. Delivering cognitive behavior therapy to young adults with symptoms of depression and anxiety using a fully automated conversational agent (Woebot): A randomized controlled trial. FMIR Mental Health 4, 2 (2017), e19.

[23] Ann Fleener. 2008. The Effects of the Literature in the Garden Curriculum on Life Skills of Children. Ph.D. Dissertation. Auburn University, Auburn, AL.

[24] Monica Frank. 2016. Depression CBT Self-Help Guide. Retrieved April 1, 2021 from https://www.excelatlife.com.

[25] Marianne Thorsen Gonzalez, Terry Hartig, Grete Grindal Patil, Egil W. Martinsen, and Marit Kirkevold. 2010. Therapeutic horticulture in clinical depression: A prospective study of active components. Fournal of Advanced Nursing 66, 9 (2010), 2002-2013. 
[26] David Gouaillier, Vincent Hugel, Pierre Blazevic, Chris Kilner, Jerome Monceaux, Pascal Lafourcade, Brice Marnier, Julien Serre, and Bruno Maisonnier. 2008. The NAO humanoid: A combination of performance and affordability. arXiv:0807.3223

[27] Bjørn Grinde and Grete Grindal Patil. 2009. Biophilia: Does visual contact with nature impact on health and wellbeing?International fournal of Environmental Research and Public Health 6, 9 (2009), 2332-2343.

[28] Jee Hyun Ha, Bumsu Chin, Doo-Heum Park, Seung-Ho Ryu, and Jaehak Yu. 2008. Characteristics of excessive cellular phone use in Korean adolescents. CyberPsychology \& Behavior 11, 6 (2008), 783-784.

[29] Heiko Hamann, Mostafa Wahby, Thomas Schmickl, Payam Zahadat, Daniel Hofstadler, Kasper Stoy, Sebastian Risi, et al. 2015. Flora robotica-mixed societies of symbiotic robot-plant bio-hybrids. In Proceedings of the 2015 IEEE Symposium Series on Computational Intelligence. IEEE, Los Alamitos, CA, 1102-1109.

[30] J. Gibson Henderson Jr. 1987. Effects of depression upon reading: A case for distinguishing effortful from automatic processes. Perceptual and Motor Skills 64, 1 (1987), 191-200.

[31] David Holstius, John Kembel, Amy Hurst, Peng-Hui Wan, and Jodi Forlizzi. 2004. Infotropism: Living and robotic plants as interactive displays. In Proceedings of the 5th Conference on Designing Interactive Systems: Processes, Practices, Methods, and Techniques. ACM, New York, NY, 215-221.

[32] Justin Hunt and Daniel Eisenberg. 2010. Mental health problems and help-seeking behavior among college students. Journal of Adolescent Health 46, 1 (2010), 3-10.

[33] M3 Information. 2018. M3 Information. Retrieved April 1, 2021 from https://www.whatsmym3.com.

[34] Neil S. Jacobson, Keith S. Dobson, Paula A. Truax, Michael E. Addis, Kelly Koerner, Jackie K. Gollan, Eric Gortner, and Stacey E. Prince. 2000. A component analysis of cognitive-behavioral treatment for depression. Fournal of Consulting and Clinical Psychology 64, 2 (2000), 295-304.

[35] Neil S. Jacobson, Christopher R. Martell, and Sona Dimidjian. 2001. Behavioral activation treatment for depression: Returning to contextual roots. Clinical Psychology: Science and Practice 8, 3 (2001), 255-270.

[36] E. Jane Costello, Alaattin Erkanli, and Adrian Angold. 2006. Is there an epidemic of child or adolescent depression? Journal of Child Psychology and Psychiatry 47, 12 (2006), 1263-1271.

[37] Nina Jøranson, Ingeborg Pedersen, Anne Marie Mork Rokstad, and Camilla Ihlebaek. 2015. Effects on symptoms of agitation and depression in persons with dementia participating in robot-assisted activity: A cluster-randomized controlled trial. Journal of the American Medical Directors Association 16, 10 (2015), 867-873.

[38] Lucy E. Keniger, Kevin J. Gaston, Katherine N. Irvine, and Richard A. Fuller. 2013. What are the benefits of interacting with nature? International fournal of Environmental Research and Public Health 10, 3 (2013), 913-935.

[39] Ronald C. Kessler, G. Paul Amminger, Sergio Aguilar-Gaxiola, Jordi Alonso, Sing Lee, and T. Bedirhan Ustun. 2007. Age of onset of mental disorders: A review of recent literature. Current Opinion in Psychiatry 20, 4 (2007), 359.

[40] Kalevi M. Korpela, Eija Stengård, and Pia Jussila. 2016. Nature walks as a part of therapeutic intervention for depression. Ecopsychology 8, 1 (2016), 8-15.

[41] Satoshi Kuribayashi, Yusuke Sakamoto, and Hiroya Tanaka. 2007. I/O plant: A tool kit for designing augmented human-plant interactions. In CHI'07 Extended Abstracts on Human Factors in Computing Systems. 2537-2542.

[42] Paul Opk Oppong Kwabena, Hanping Mao, and Lin Li. 2018. New type Arduino plant grower (GCKJ). In Proceedings of the 2018 ASABE Annual International Meeting. 1.

[43] Leib Litman, Jonathan Robinson, and Tzvi Abberbock. 2017. TurkPrime.com: A versatile crowdsourcing data acquisition platform for the behavioral sciences. Behavior Research Methods 49, 2 (2017), 433-442.

[44] Donald Meichenbaum, Jon Carlson, and Diane Kjos. 2007. Cognitive-Behavioral Therapy. American Psychological Association.

[45] Sanika Moharana, Alejandro E. Panduro, Hee Rin Lee, and Laurel D. Riek. 2019. Robots for joy, robots for sorrow: Community based robot design for dementia caregivers. In Proceedings of the 2019 14th ACM/IEEE International Conference on Human-Robot Interaction (HRI'19). IEEE, Los Alamitos, CA, 458-467.

[46] Ramin Mojtabai, Mark Olfson, and Beth Han. 2016. National trends in the prevalence and treatment of depression in adolescents and young adults. Pediatrics 138, 6 (2016), e20161878.

[47] Moodlytics. 2014. Moodlytics. Retrieved April 1, 2021 from http://www.moodlytics.com.

[48] Akira Nakayasu. 2010. Himawari: Shape memory alloy motion display for robotic representation. In CHI'10 Extended Abstracts on Human Factors in Computing Systems. ACM, New York, NY, 4327-4332.

[49] Michelle G. Newman, Lauren E. Szkodny, Sandra J. Llera, and Amy Przeworski. 2011. A review of technology-assisted self-help and minimal contact therapies for anxiety and depression: Is human contact necessary for therapeutic efficacy? Clinical Psychology Review 31, 1 (2011), 89-103.

[50] World Health Organization. 2012. Depression in Europe: Facts and figures. Retrieved April 1, 2021 from http:// www.euro.who.int/en/health-topics/noncommunicable-diseases/mental-health/news/news/2012/10/depression-ineurope/depression-in-europe-facts-and-figures. 
[51] Ruth K. Raanaas, Katinka Horgen Evensen, Debra Rich, Gunn Sjøstrøm, and Grete Patil. 2011. Benefits of indoor plants on attention capacity in an office setting. Journal of Environmental Psychology 31, 1 (2011), 99-105.

[52] Mary Redmayne, Euan Smith, and Michael J. Abramson. 2013. The relationship between adolescents' well-being and their wireless phone use: A cross-sectional study. Environmental Health 12, 1 (2013), 90.

[53] David A. Richards, David Ekers, Dean McMillan, Rod S. Taylor, Sarah Byford, Fiona C. Warren, Barbara Barrett, et al. 2016. Cost and outcome of behavioural activation versus cognitive behavioural therapy for depression (COBRA): A randomised, controlled, non-inferiority trial. Lancet 388, 10047 (2016), 871-880.

[54] Darius A. Rohani, Andrea Quemada Lopategui, Nanna Tuxen, Maria Faurholt-Jepsen, Lars V. Kessing, and Jakob E. Bardram. 2020. MUBS: A personalized recommender system for behavioral activation in mental health. In Proceedings of the 2020 CHI Conference on Human Factors in Computing Systems. 1-13.

[55] Laura Kann, Tim McManus, William A. Harris, Shari L. Shanklin, Katherine H. Flint, Joseph Hawkins, Barbara Queen, et al. 2016. Youth risk behavior surveillance-United States. Morbidity and Mortality Weekly Report Surveillance Summaries 65, 6 (2015), 1-168. Retrieved April 1, 2021 from https://www.cdc.gov/healthyyouth/data/yrbs/pdf/ 2015/ss6506_updated.pdf.

[56] Selma Šabanović, Wan-Ling Chang, Casey C. Bennett, Jennifer A. Piatt, and David Hakken. 2015. A robot of my own: Participatory design of socially assistive robots for independently living older adults diagnosed with depression. In Proceedings of the International Conference on Human Aspects of IT for the Aged Population. 104-114.

[57] Pedro Sanches, Axel Janson, Pavel Karpashevich, Camille Nadal, Chengcheng Qu, Claudia Daudén Roquet, Muhammad Umair, et al. 2019. HCI and affective health: Taking stock of a decade of studies and charting future research directions. In Proceedings of the 2019 CHI Conference on Human Factors in Computing Systems. 1-17.

[58] Mercedes Sánchez-Martínez and Angel Otero. 2009. Factors associated with cell phone use in adolescents in the community of Madrid (Spain). CyberPsychology \& Behavior 12, 2 (2009), 131-137.

[59] Harpreet Sareen and Pattie Maes. 2019. Cyborg botany: Exploring in-planta cybernetic systems for interaction. In Extended Abstracts of the 2019 CHI Conference on Human Factors in Computing Systems. ACM, New York, NY, LBW0237.

[60] Nicola S. Schutte, Navjot Bhullar, Emma J. Stilinović, and Katheryn Richardson. 2017. The impact of virtual environments on restorativeness and affect. Ecopsychology 9, 1 (2017), 1-7.

[61] Bijo Sebastian, Dip Narayan Ray, and Somajyoti Majumder. 2015. Design and analysis of a tree climbing robot. In Proceedings of the 2015 Conference on Advances in Robotics. ACM, New York, NY, 17.

[62] Jinsil Hwaryoung Seo, Annie Sungkajun, and Jinkyo Suh. 2015. Touchology: Towards interactive plant design for children with autism and older adults in senior housing. In Proceedings of the 33rd Annual ACM Conference Extended Abstracts on Human Factors in Computing Systems. 893-898.

[63] Eleni Stavrinidou, Roger Gabrielsson, Eliot Gomez, Xavier Crispin, Ove Nilsson, Daniel T. Simon, and Magnus Berggren. 2015. Electronic plants. Science Advances 1, 10 (2015), e1501136.

[64] Helene Steiner, Paul Johns, Asta Roseway, Chris Quirk, Sidhant Gupta, and Jonathan Lester. 2017. Project Florence: A plant to human experience. In Proceedings of the 2017 CHI Conference Extended Abstracts on Human Factors in Computing Systems. 1415-1420.

[65] Masahiro Toyoda, Yuko Yokota, Marni Barnes, and Midori Kaneko. 2020. Potential of a small indoor plant on the desk for reducing office workers' stress. HortTechnology 30, 1 (2020), 55-63.

[66] Agnes E. Van Den Berg and Mariëtte H. G. Custers. 2011. Gardening promotes neuroendocrine and affective restoration from stress. fournal of Health Psychology 16, 1 (2011), 3-11.

[67] Lex Van Velsen, Jobke Wentzel, and Julia E. W. C. Van Gemert-Pijnen. 2013. Designing eHealth that matters via a multidisciplinary requirements development approach. FMIR Research Protocols 2, 1 (2013), e21.

[68] Kazuyoshi Wada, Takanori Shibata, Takashi Asada, and Toshimitsu Musha. 2007. Robot therapy for prevention of dementia at home. fournal of Robotics and Mechatronics 19, 6 (2007), 691.

[69] Alex C. Williams, Harmanpreet Kaur, Gloria Mark, Anne Loomis Thompson, Shamsi T. Iqbal, and Jaime Teevan. 2018. Supporting workplace detachment and reattachment with conversational intelligence. In Proceedings of the $2018 \mathrm{CHI}$ Conference on Human Factors in Computing Systems. 1-13.

[70] Kirsten Windfuhr, David While, Isabelle Hunt, Pauline Turnbull, Rebecca Lowe, Jimmy Burns, Nicola Swinson, et al. 2008. Suicide in juveniles and adolescents in the United Kingdom. Journal of Child Psychology and Psychiatry 49, 11 (2008), 1155-1165.

Received May 2020; revised September 2020; accepted December 2020 